\title{
Near infrared spectroscopy to study the brain: an overview
}

\author{
M. WOLF*', G. MORREN, D. HAENSSE, T. KAREN, U. WOLF, J.C. FAUCHÈRE, \\ and H.U. BUCHER
}

Clinic of Neonatology, University Hospital Zurich,

Frauenklinikstr. 10, 8091 Zurich, Switzerland

This paper gives an overview of principles, technologies, and applications using near infrared spectrometry and imaging (NIRS and NIRI) to study brain function. The physical background is reviewed and technologies and their properties are discussed. Advantages and limitations of NIRI are described. The basic functional signals obtained by NIRI, the neuronal and the hemodynamic signal are described and in particular publications about the former are reviewed. Applications in adults and neonates are reviewed, too.

Keywords: near infrared spectrometry, near infrared imaging, functional activation.

\section{Physical consideration of near infrared spectroscopy and imaging}

Near-infrared light penetrates tissue relatively deeply and easily passes through the skull and brain. To analyze brain function a sensor emitting near-infrared light is placed on the surface of the head and at a few centimetres distance the intensity of the reflected light is detected. This light intensity depends on the optical properties of the brain, e.g., if there is an increase in blood concentration in the brain, less light will pass and the intensity at the detector will be reduced. The changes associated with brain activity can non-invasively be detected using near-infrared spectrophotometry (NIRS) and imaging (NIRI).

Tissue can be optically characterized by two parameters, scattering and absorption. Absorption reduces the light transmission and constitutes a spectrum, which is a finger print of a specific substance. An important aim of NIRI is to interrogate deep layers of tissue. Below $650 \mathrm{~nm}$ oxyhemoglobin $\left(\mathrm{O}_{2} \mathrm{Hb}\right)$ and deoxyhemoglobin $(\mathrm{HHb})$ and above $950 \mathrm{~nm}$ water absorb strongly. Therefore, NIRI uses wavelengths between $650 \mathrm{~nm}$ and $950 \mathrm{~nm}$, where light penetrates tissue relatively deeply and $\mathrm{O}_{2} \mathrm{Hb}, \mathrm{HHb}$, cytochrome aa3, lipids and water show distinct spectra.

Characteristic for light propagation in tissues is the strong scattering. In the NIR range, the scattering coefficient is more than one order of magnitude higher than the absorption coefficient and light propagation is disordered after 1-mm path. Therefore, NIRI depends on adequate theoretical models, such as, e.g., the diffusion approximation to the Boltzmann transport equation for the semi-infinite boundary condition [1-8].

*e-mail: martin.wolf@usz.ch
The basic measuring scheme is the following. A light source is placed on the intact head. At a distance of 2-5 $\mathrm{cm}$, a light detector receives the reemerging light. The photons travelling from source to detector penetrate several $\mathrm{cm}$ deep into tissue and contain the information about the brain [9-12].

For imaging applications, a mesh of sources and detectors is placed on the region of interest. The measured data is spatially reconstructed to obtain images of absorption and scattering. 2D (here called NIRI or optical imaging, also called optical topography or diffuse optical imaging) and 3D (optical tomography) images can thus be obtained (overview of the technical approaches in adults and neonates $[13,14]$, summaries of functional imaging studies in adults [15-20]). Optical imaging is used by an increasing number of research groups [13]. It has proven to be advantageous compared to measurements on single locations, because changes can be localized and thus, measurements in single locations may not be representative for a larger volume of tissue, which is particularly true when functionally studying the brain. Online display of optical images visualizing $\mathrm{O}_{2} \mathrm{Hb}$ and $\mathrm{HHb}$ concentration with a time resolution of up to $6 \mathrm{~Hz}$ have been reported [21].

One major effort in the development of 3D optical tomography is the detection of cancer in the female breast [22-24]]. Time domain optical tomography was applied to study the head of neonates $[13,14,25]$. 3D optical tomography still has a low spatial resolution of 1 to $2 \mathrm{~cm}$ and requires 10 min of time to acquire an image [13] and was despite this limit recently used for a functional study [14,26]. However, the tomographic approach's time resolution severely limits its application for functional studies of the brain, but 2D optical imaging is feasible also for neuronal activity. 


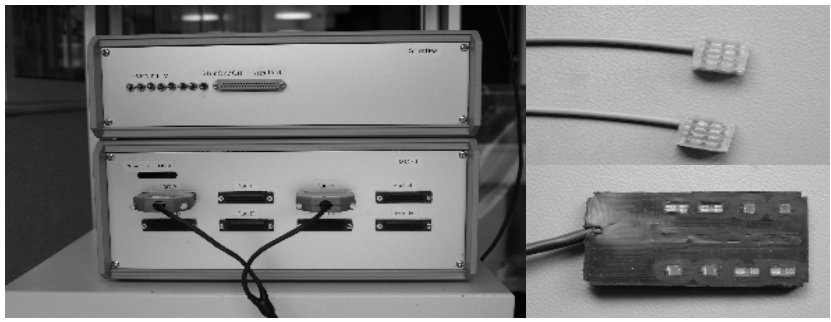

Fig. 1. Left: MCPII measuring system (bottom) and stimulation unit (top). Right: LEDs for visual stimulation (top right). The sensor contains 4 light sources (each with $730 \mathrm{~nm}$ and $830 \mathrm{~nm}$ LEDs) and 4 detectors (photodiodes), covering an area of 2.5 by $3.75 \mathrm{~cm}$ with 10 light bundles (bottom right).

Recently, we have built a continuous wave imaging device with a $100-\mathrm{Hz}$ time resolution, which can measure parts of the brain [27]. This versatile, multi-channel NIRS instrument (Fig. 1) for the purpose of mapping brain activation in the neonatal and adult brain in response to motor, tactile, and visual stimulation. The optical linearity, stability, and high signal to noise ratio $(>70 \mathrm{~dB})$ of the instrument were demonstrated using an in vitro validation procedure. It has 48 channels, $100-\mathrm{Hz}$ time resolution and a dual 16-bit AD-converter. The set-up depends on the sensors used, typically it contains 4-light source location with 3 wavelengths of $730 \mathrm{~nm}, 800 \mathrm{~nm}$, and $850 \mathrm{~nm}$ and 4 detector locations. This instrument has been miniaturized in the mean time and there is a wireless sensor available (Fig. 2).

NIRI currently has the following limitations:

- only the outer cortex $(25 \mathrm{~mm})$ can be investigated in adults, the head of neonates can be transilluminated, however. The skull is relatively transparent to near-infrared light,

- spatial resolution is limited ( 10 mm), therefore usually no anatomical information is available,

- extracerebral blood flow may influence the results, special techniques (multidistance) remove this effect,

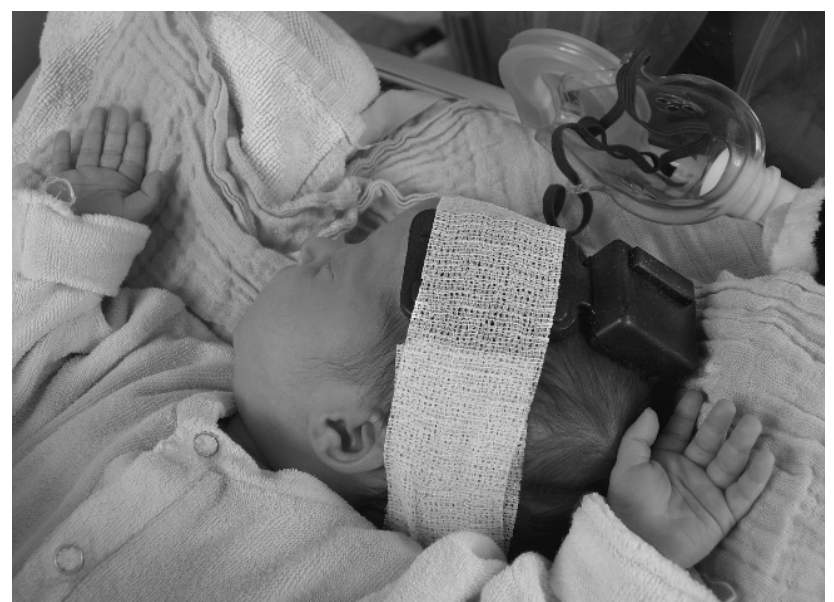

Fig. 2. Wireless NIRI instrument. The advantage of wireless technology is that it is more comfortable and less susceptible to movement articats, because there is no cable, which pulls the sensor. The device only weighs $40 \mathrm{~g}$ and the battery lasts for approximately $3 \mathrm{~h}$.
- motion artifacts lead to changes in the signals and have to be eliminated.

NIRI has become a quickly growing method to study brain for the following reasons:

- NIRI quantifies important physiological measures, $\mathrm{O}_{2} \mathrm{Hb}, \mathrm{HHb}$, tissue oxygenation, cytochrome aa3 (an enzyme of the respiration chain), $\mathrm{H}_{2} \mathrm{O}$ and lipids,

- the technique is non-invasive and painless,

- non-ionizing radiation is used,

- it can be used at the bedside,

- probes are attached relatively quickly to a subject,

- no sedation of the patients is required,

- NIRI measures continuously and displays results in real time,

- long term measurements are feasible (monitoring),

- NIRI is immune to electro-magnetic interference,

- NIRI is relatively inexpensive.

\section{Brain studies by near-infrared imaging}

One of the most important applications of NIRI is to functionally study activity of the brain and its development. Two major types of optical signals associated with brain activity can be detected by NIRI: The neuronal and the hemodynamic signal.

\subsection{Neuronal signal}

One potentially very interesting discovery is the detectability of the neuronal signal by NIRI, which is related to optical changes directly associated with neuronal activity. This signal arises within $100 \mathrm{~ms}$ after the onset of the stimulation and has been studied at the cellular level, in animal studies and in humans.

At the cellular level, it is, according to the literature, certain that the optical properties of neurons change depending on the activity. Several types of optical changes at the neuron have been suggested. Extensive reviews can be found in Refs. 15 and 28-31. Here is a short overview of the effects found.

Several authors have located optical changes in the neuronal membrane. Cohen [28] found voltage dependent changes in birefringence in the membrane of the axon of nerve cells. He suggested that the observed effect was either due to a thinning of the membrane or the Kerr effect. Stepnoski [32] observed a similar signal, which was practically simultaneous to the action potential. Tasaki [30] found that thermal (heat production and absorption), mechanical (swelling and shrinking) and optical (birefringence) changes in the axon were simultaneous and related to a sudden swelling of the gel layer of the membrane due to an increase in water content. This gel layer is superficial and about $0.5-\mu \mathrm{m}$ thick and an integral part of the membrane of the axon.

Another type of optical change was located at the nerve terminal. Salzberg [33] studied the intact neurohypophysis of mice and reported large and rapid decreases in light scat- 
tering, which accompanied the secretion by nerve terminals. These changes occurred in two phases and the fractional light intensity change was approximately $0.2 \%$.

As a third type of signal, Cohen [28] reported a biphasic change in light scattering $\left(90^{\circ}\right)$, i.e., an increase in light scattering that was simultaneous to the action potential, after a return to the baseline another increase in light scattering was observed with a peak after $20 \mathrm{~ms}$.

Sable [31] indicates other possible effects, such as cell swelling, changes in the cytoskeleton and changes in the geometry of microtubules, which change the light scattering of neuronal tissue.

In conclusion, there is extensive literature on studies in single neurons and brain slices, which demonstrates several effects as the origin of the neuronal signal at the neuronal level.

Further studies were performed on the exposed cortex in animals [31]. Grinvald [34] reported a decrease in reflected light $200 \mathrm{~ms}$ after the onset of whisker stimulation. Rector [35] found a similar response synchronous to the electrical signal. Malonek [36] reported an increase in light intensity with a latency (i.e., the period between stimulation and response) of $200 \mathrm{~ms}$.

Rector [37] found four temporal components of the brain activity in rats. A weak response with a peak at $30 \mathrm{~ms}$ after the stimulus correlating with presynaptic activation was followed by an opposite and much larger signal at $80 \mathrm{~ms}$. These two early responses were limited in their spatial distribution and were directly related to neural activity. The two slower components with peaks at $300 \mathrm{~ms}$ and $800 \mathrm{~ms}$ were spatially more extended and overlapped. The signal at 300 ms appeared to be consistent with the hemodynamic mapping signal described by Malonek [36], which is also called "the dip", i.e., a short increase in $\mathrm{HHb}$ at the onset of functional stimulation, which occurs before a marked decrease in $\mathrm{HHb}$ due to an increase in blood flow at $800 \mathrm{~ms}$, which is the hemodynamic signal described in below. Thus, the neuronal signal is detectable in the exposed cortex.

Several research laboratories have therefore tried to detect the neuronal signal non-invasively in human subjects. The first attempt was published by Gratton in 1995. The group of Gratton continued to be productive in this field [38-51]. The reported neuronal signal is an increase in the phase of a frequency domain NIRI instrument.

The research group of the Berlin Neuroimaging Centre at the Charite was not able to reproduce the results of Gratton [52] (Please note that this paper is based on the first work of the Berlin Neuroimaging Centre and is therefore cited first. It was already presented at a conference in 1999 and suffered a delay in publication). Furthermore, Syré [52] performed Monte Carlo simulations to support their results and found that the expected change due to the neuronal signal was $0.01 \%$ in intensity or 0.01 ps time of flight (or $0.0004^{\circ}$ in phase), which was much lower than the change reported by Gratton. The simulated change in time of flight was below the limit of detectability. From this simulation, it seemed to be more promising to study changes in intensity than in time of flight. Later indeed, a decrease in light intensity by $0.05 \%$ associated with neuronal activity was detected [53]. This signal was observed in 5 subjects. Movement artifacts were excluded, because the change was visible also when using median nerve stimulation below the motor threshold. Steinbrink [53] refers again to the Monte Carlo simulation and states, that the expected magnitude of the intensity change was $0.02 \%$. Obrig [15] revealed that the Berlin Neuroimaging Centre no longer trusted its own results [53]. Since the signal was not found in the visual cortex or during stimulation below the motor threshold, the previous data were attributed to movement artifacts. Later, Steinbrink provided more details and confirmed that his previous results were due to movement artefacts [54]. No neuronal signal was found in the visual cortex. The findings of a new simulation were reported in addition. The maximum expected change was $0.01 \%$ in light intensity and $0.01 \mathrm{ps}$ in time of flight, but more likely to be $0.0001 \%$. This wide range of the simulated data shows that their Monte Carlo model is not reliable, because it depends on too many assumptions. This is not surprising since many factors are not precisely known. The conclusion was that it is not feasible to detect the neuronal signal non-invasively.

In our experiments [55-57], we found a mean change of light intensity of $0.0056 \%$ and 0.12 ps in time of flight (only one significant signal) in the motor cortex [55] during finger tapping and a mean change of light intensity of $\sim 0.05 \%$ and no significant changes in time of flight in the visual cortex [56]. An independent component analysis was more sensitive to the neuronal signal, i.e., significant intensity changes were detected in 9 out of 14 subjects and were visible even in time frequency plots [57].

In order to distinguish neuronal signals from noise or artefacts, the following criteria were established.

The signal had to be:

- significantly different from noise $(\mathrm{p}<0.001)$,

- related to the stimulation signal (tapping or reversing), i.e., disappears during rest periods,

- localized to exclude movement artifacts.

In addition there was good agreement between the latencies.

Franceschini [58] detected a change in intensity due to neuronal activity in $43 \%$ of the measurements during finger tapping, 60\% during tactile stimulation and 23\% during median nerve stimulation. The amplitude of the intensity change was $\sim 0.1 \%$. Franceschini only measured light intensity and not time of flight, but measured at two wavelengths. They also carried out numerical simulations. In addition, they further developed criteria to assess the robustness of the detected signal.

The signal had to be:

- similar between the two wavelengths,

- significantly different between stimulation and rest,

- similar between subsets of the data,

- lower in amplitude on the ipsilateral side compared to the contralateral side of the brain

- localized. 
NIRI was compared to EEG by Medvedev in an object detection task using an independent component analysis. The good agreement is reported in Ref. 59.

A further group detected neuronal signals during a study on preattentive changes [60]. There are no reports on studies in neonates so far. Thus, it has not been tested, whether the neuronal signal is visible in neonates.

As it can be seen from this review of the literature, there is some controversy, whether the neuronal activity can be detected in non-invasively in adult human subjects. However, evidence accumulates that the neuronal signal can be detected. This may potentially be very exciting, because the neuronal signal is very likely to be more localized and thus, will deliver more precise information about activation and neurovascular coupling can be studied in more detail.

\subsection{Hemodynamic signal}

The hemodynamic signal is related to an increased blood perfusion in an area of activated neurons due to neurovascular coupling. Brain activity due to a specific stimulation leads to an increase in the local oxygen consumption immediately followed by an increase in blood flow [61], which in turn changes the oxygenation and hemoglobin concentrations, i.e., an increase in $\mathrm{O} \mathrm{Hb}$ and a decrease in $\mathrm{HHb}$. These changes occur within a few seconds after the onset of the stimulation, are reproducible [62,63], highly localized [64] and have been extensively studied in adults [15-20,65].

There are few reports in the literature on hemodynamic effects in neonates, visual cortex [66-68], auditory cortex [69-72], speech frontal cortex [73], olfactory stimulation [74,75], pain [76-78], and motor cortex [14]. Bartocci's [75] findings during the presentation of unpleasant smells to infants are highly particular, because a decrease in $\mathrm{O} \mathrm{Hb}$ concentration was observed, while the $\mathrm{HHb}$ concentration remained approximately constant. This implies that the oxygenation of the brain dropped in the presence of an unpleasant odour. Kotilahti [72] found that the hemodynamic changes depend on the sleep state. The other studies can be summarized that as in adults, the $\mathrm{O}_{2} \mathrm{Hb}$ concentration increases a few seconds after the onset of the stimulation. A typical examples of an ensemble average is displayed in Fig. 3 [68]. In contrast to the findings in adults, the $\mathrm{HHb}$ concentration increases in the majority of the infants $(90 \%$ in Ref. 79, 60.7\% in Ref. 70, 61.5\% in Ref. 71), no clear changes [69] or remains unchanged [72]. This may imply that the additional oxygen consumption due to neuronal activity is not compensated by the blood supply to the same degree as in adults. Furthermore, it clearly demonstrates that signals such as the BOLD signal, which exclusively reflect the $\mathrm{HHb}$, are not sufficient to do functional studies in infants. Brain activity may be missed due to absent changes in $\mathrm{HHb}$ concentration, but could be detected by a method, which is sensitive to $\mathrm{O}_{2} \mathrm{Hb}$ concentration changes.

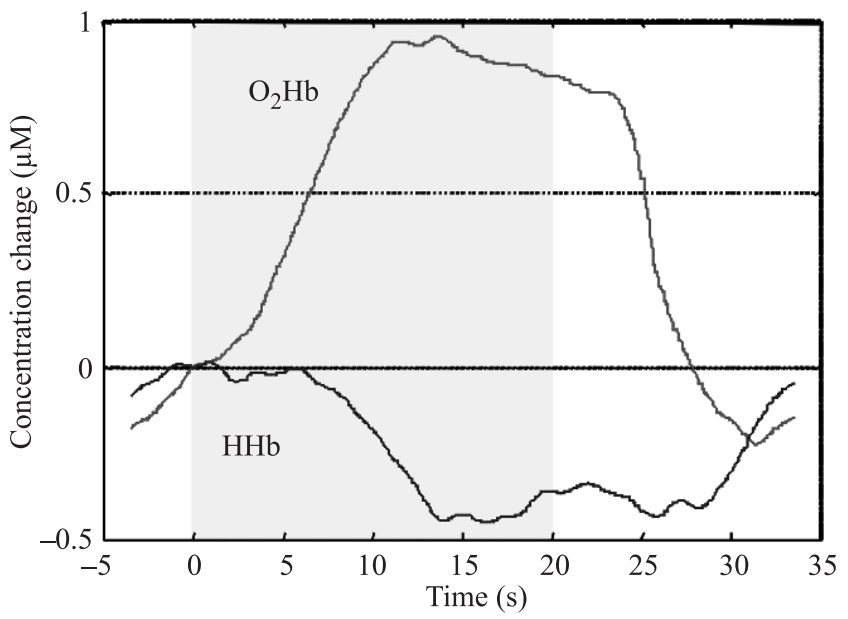

Fig. 3. The ensemble average of all significant hemdynamic responses $\left(\mathrm{O}_{2} \mathrm{Hb}\right.$ and $\left.\mathrm{HHb}\right)$ to stimulation in the visual cortex. After the onset of the stimulation $\mathrm{O}_{2} \mathrm{Hb}$ increases and the $\mathrm{HHb}$ decreases until both components reach a plateau. The end of the stimulation is followed by a decrease in $\mathrm{O}_{2} \mathrm{Hb}$ and an increase in $\mathrm{HHb}$ until they return to baseline. The decrease in $\mathrm{HHb}$ is approximately twice smaller than the increase in $\mathrm{O}_{2} \mathrm{Hb}$. There is a delay in the response of the $\mathrm{HHb}$ compared to the $\mathrm{O}_{2} \mathrm{Hb}$, which was not seen in adult subjects.

Optical imaging techniques in neonates clearly demonstrate a diagnostic potential [68,69,72,80-83], while optical tomography [14], although promising and fascinating, still needs development.

Chen [69] found that the auditory functional response of healthy infants (both $\mathrm{O}_{2} \mathrm{Hb}$ and total hemoglobin $(\mathrm{tHb})$ increase) is highly significantly different from the one of infants suffering from hypoxic-ischemic encephalopathy (in a majority of the infants both $\mathrm{O}_{2} \mathrm{Hb}$ and tHb decrease). This paper clearly demonstrates the potential of NIRI to assess and understand the effects of brain injury and possibly predict outcome.

\section{Conclusions}

NIRI has been increasingly applied to study the brain and found to be reliable and reproducible in terms of hemodynamic effects associated with brain activity. Whether neuronal activity can be detected non-invasively is still somewhat controversial, although evidence in favour is accumulating. Due to its excellent properties for clinical applications, it is expected that the use NIRI in medicine will increase tremendously in the near future.

\section{References}

1. S.R. Arridge, M. Cope, and D.T. Delpy, "The theoretical basis for the determination of optical pathlengths in tissue: temporal and frequency analysis", Phys. Med. Biol. 37, 1531-1560 (1992).

2. M.S. Patterson, B. Chance, and B.C. Wilson, "Time resolved reflectance and transmittance for the non-invasive 
measurement of tissue optical properties", Appl. Optics 28, 2331-2336 (1989).

3. D.A. Boas, M.A. O'Leary, B. Chance, and A.G. Yodh, "Scattering of diffuse photon density waves by spherical inhomogeneities within turbid media: analytical solution and applications", Proc. Natl. Acad. Sci. USA 91, 4887-4891 (1994).

4. S. Wray, M. Cope, D.T. Delpy, J.S. Wyatt, and E.O. Reynolds, "Characterization of the near infrared absorption spectra of cytochrome aa3 and haemoglobin for the non-invasive monitoring of cerebral oxygenation", Biochim. Biophys. Acta 933, 184-192 (1988).

5. S. Matcher, P. Kirkpatrick, K. Nahid, M. Cope, and D.T. Delpy, "Absolute quantification methods in tissue near infrared spectroscopy", Proc. SPIE 2389, 486-495 (1995).

6. P. van der Zee, M. Cope, S.R. Arridge, M. Essenpreis, L.A. Potter, A.D. Edwards, J.S. Wyatt, D.C. McCormick, S.C. Roth, and E.O. Reynolds, "Experimentally measured optical pathlengths for the adult head, calf and forearm and the head of the newborn infant as a function of inter optode spacing", Adv. Exp. Med. Biol. 316, 143-153 (1992).

7. S. Fantini, M.A. Franceschini-Fantini, J.S. Maier, S.A. Walker, B. Barbieri, and E. Gratton, "Frequency-domain multichannel optical detector for noninvasive tissue spectroscopy and oximetry", Opt. Eng. 34, 32-42 (1995).

8. M.A. O'Leary, D.A. Boas, B. Chance, and A.G. Yodh, "Experimental images of heterogeneous turbid media by frequency-domain diffusing-photon tomography", Opt. Lett. 20, 426-428 (1995).

9. S. Eda and E. Okada, "Monte Carlo analysis of time-resolved spatial sensitivity profiles in realistic head models", Proc. SPIE 4250, 383-390 (2001).

10. V. Toronov, A. Webb, J.H. Choi, M. Wolf, A. Michalos, E. Gratton, and D. Hueber, "Investigation of human brain hemodynamics by simultaneous near-infrared spectroscopy and functional magnetic resonance imaging", Med. Phys. 28, 521-527 (2001).

11. J.H. Choi, M. Wolf, V.Y. Toronov, A. Michalos, and E. Gratton, "Spatio-temporal analysis of the cerebral spontaneous oscillation”, Proc. SPIE 5330, 29-37 (2004).

12. E. Ohmae, Y. Ouchi, M. Oda, T. Suzuki, S. Nobesawa, T. Kanno, E. Yoshikawa, M. Futatsubashi, Y. Ueda, H. Okada, and Y. Yamashita, "Cerebral hemodynamics evaluation by near-infrared time-resolved spectroscopy: correlation with simultaneous positron emission tomography measurements", Neuroimage 29, 697-705 (2006).

13. J.C. Hebden, "Advances in optical imaging of the newborn infant brain", Psychophysiol. 40, 501-510 (2003).

14. J.C. Hebden and T. Austin, "Optical tomography of the neonatal brain”, Eur. Radiol. 17, 2926-2933 (2007).

15. H. Obrig and A. Villringer, "Beyond the visible-imaging the human brain with light", J. Cereb. Blood. Flow. Metab. 23, 1-18 (2003).

16. J. Steinbrink, A. Villringer, F. Kempf, D. Haux, S. Boden, and H. Obrig, "Illuminating the BOLD signal: combined fMRI-fNIRS studies", Magn. Reson. Imaging 24, 495-505 (2006).

17. Y. Hoshi, "Functional near-infrared spectroscopy: potential and limitations in neuroimaging studies", Int. Rev. Neurobiol. 66, 237-266 (2005).
18. Y. Hoshi, "Functional near-infrared optical imaging: utility and limitations in human brain mapping", Psychophysiol. 40, 511-520 (2003).

19. E. Gratton, V. Toronov, U. Wolf, M. Wolf, and A. Webb, "Measurement of brain activity by near-infrared light", $J$. Biomed. Opt. 10, 11008 (2005).

20. M. Wolf, M. Ferrari, and V. Quaresima, "Progress of near-infrared spectroscopy and topography for brain and muscle clinical applications", J. Biomed. Opt. 12, 062104 (2007).

21. M.A. Franceschini, V. Toronov, M.E. Filiaci, E. Gratton, and S. Fantini, "On-line optical imaging of the human brain with 160-ms temporal resolution”, Opt. Express 6, 49 (2000).

22. S. Fantini and P. Taroni, "Optical Mammography", in Cancer Imaging: Lung and Breast Carcinomas, pp. 449-458, edited by M.A. Hayat, Elsevier, 2007.

23. R.X. Xu and S.P. Povoski, "Diffuse optical imaging and spectroscopy for cancer”, Expert Rev. Med. Devic. 4, 83-95 (2007).

24. D.R. Leff, O.J. Warren, L.C. Enfield, A. Gibson, T. Athanasiou, D.K. Patten, J. Hebden, G.Z. Yang, and A. Darzi, "Diffuse optical imaging of the healthy and diseased breast: A systematic review", Breast Cancer Res. Tr. 108, 9-22 (2007).

25. D.A. Benaron, D.C. Ho, S. Spilman, J.P. Van Houten, and D.K. Stevenson, "Tomographic time-of-flight optical imaging device", Adv. Exp. Med. Biol. 361, 207-14 (1994).

26. T. Austin, "Optical imaging of the neonatal brain", Arch. Dis. Child.-Fetal 92, F238-41 (2007).

27. D. Haensse, P. Szabo, D. Brown, J.C. Fauchere, P. Niederer, H.U. Bucher, and M. Wolf, "New multichannel near infrared spectrophotometry system for functional studies of the brain in adults and neonates", Opt. Express 13, 4525-4538 (2005).

28. L.B. Cohen, "Changes in neuron structure during action potential propagation and synaptic transmission", Physiol. Rev. 53, 373-418 (1973).

29. D.W. Hochman, "Intrinsic optical changes in neuronal tissue. Basic mechanisms", Neurosurg. Clin. N. Am. 8, 393-412 (1997).

30. I. Tasaki, "Rapid structural changes in nerve fibers and cells associated with their excitation processes", Jpn. J. Physiol. 49, 125-138 (1999).

31. J.J. Sable, D.M. Rector, and G. Gratton, "Optical neurophysiology based on animal models", IEEE Eng. Med. Biol. 26, 17-24 (2007).

32. R.A. Stepnoski, A. LaPorta, F. Raccuia-Behling, G.E. Blonder, R.E. Slusher, and D. Kleinfeld, "Noninvasive detection of changes in membrane potential in cultured neurons by light scattering", Proc. Natl. Acad. Sci. USA 88, 9382-9386 (1991).

33. B.M. Salzberg, A.L. Obaid, and H. Gainer, "Large and rapid changes in light scattering accompany secretion by nerve terminals in the mammalian neurohypophysis", $J$. Gen. Physiol. 86, 395-411 (1985).

34. A. Grinvald, E. Lieke, R.D. Frostig, C.D. Gilbert, and T.N. Wiesel, "Functional architecture of cortex revealed by optical imaging of intrinsic signals", Nature 324, 361-364 (1986).

35. D.M. Rector, G.R. Poe, M.P. Kristensen, and R.M. Harper, "Light scattering changes follow evoked potentials from hippocampal Schaeffer collateral stimulation", J. Neurophysiol. 78, 1707-1713 (1997). 
36. D. Malonek and A. Grinvald, "Interactions between electrical activity and cortical microcirculation revealed by imaging spectroscopy: implications for functional brain mapping", Science 272, 551-554 (1996).

37. D.M. Rector, R.F. Rogers, J.S. Schwaber, R.M. Harper, and J.S. George, "Scattered-light imaging in vivo tracks fast and slow processes of neurophysiological activation", Neuroimage 14, 977-994 (2001).

38. M.C. DeSoto, M. Fabiani, D.C. Geary, and G. Gratton, "When in doubt, do it both ways: brain evidence of the simultaneous activation of conflicting motor responses in a spatial stroop task", J. Cognitive Neurosci. 13, 523-536 (2001).

39. G. Gratton, P.M. Corballis, E. Cho, M. Fabiani, and D.C. Hood, "Shades of grey matter: noninvasive optical images of human brain responses during visual stimulation", Psychophysiol. 32, 505-509 (1995).

40. G. Gratton, M. Fabiani, P.M. Corballis, D.C. Hood, M.R. Goodman-Wood, J. Hirsch, K. Kim, D. Friedman, and E. Gratton, "Fast and localized event-related optical signals (EROS) in the human occipital cortex: comparisons with the visual evoked potential and fMRI", Neuroimage 6, 168-180 (1997).

41. G. Gratton, M. Fabiani, P.M. Corballis, and E. Gratton, "Noninvasive detection of fast signals from the cortex using frequency-domain optical methods", Ann. NY. Acad. Sci. 820, 286-298 (1997).

42. G. Gatton, M. Fabiani, and P.M. Corballis, "Can we measure correlates of neuronal activity with non-invasive optical methods?", Adv. Exp. Med. Biol. 413, 53-62 (1997).

43. G. Gratton, A. Sarno, E. Maclin, P.M. Corballis, and M. Fabiani, "Toward noninvasive 3-D imaging of the time course of cortical activity: investigation of the depth of the event-related optical signal", Neuroimage 11, 491-504 (2000).

44. G. Gratton and M. Fabiani, "The event-related optical signal: a new tool for studying brain function", Int. J. Psychophysiol. 42, 109-121 (2001).

45. G. Gratton and M. Fabiani, "Shedding light on brain function: the event-related optical signal", Trends. Cogn. Sci. 5, 357-363 (2001).

46. G. Gratton and M. Fabiani, "The event-related optical signal (EROS) in visual cortex: replicability, consistency, localization, and resolution", Psychophysiol. 40, 561-571 (2003).

47. K.A. Low, E. Leaver, A.F. Kramer, M. Fabiani, and G. Gratton, "Fast optical imaging of frontal cortex during active and passive oddball tasks", Psychophysiol. 43, 127-136 (2006).

48. G. Gratton, C.R. Brumback, B.A. Gordon, M.A. Pearson, K.A. Low, and M. Fabiani, "Effects of measurement method, wavelength, and source-detector distance on the fast optical signal", Neuroimage 32, 1576-1590 (2006).

49. E.L. Maclin, K.A. Low, J.J. Sable, M. Fabiani, and G. Gratton, "The event-related optical signal to electrical stimulation of the median nerve", Neuroimage 21, 1798-1804 (2004).

50. E.L. Maclin, K.A. Low, M. Fabiani, and G. Gratton, "Improving the signal-to-noise ratio of event-related optical signals", IEEE Eng. Med. Biol. Mag. 26, 47-51 (2007).

51. T. Rinne, G. Gratton, M. Fabiani, N. Cowan, E. Maclin, A. Stinard, J. Sinkkonen, K. Alho, and R. Naatanen, "Scalp- -recorded optical signals make sound processing in the auditory cortex visible?", Neuroimage 10, 620-624 (1999).

52. F. Syre, H. Obrig, J. Steinbrink, M. Kohl, R. Wenzel, and A. Villringer, "Are VEP correlated fast optical signals detectable in the human adult by non-invasive nearinfrared spectroscopy (NIRS)?”, Adv. Exp. Med. Biol. 530, 421-431 (2003).

53. J. Steinbrink, M. Kohl, H. Obrig, G. Curio, F. Syre, F. Thomas, H. Wabnitz, H. Rinneberg, and A. Villringer, "Somatosensory evoked fast optical intensity changes detected non-invasively in the adult human head", Neurosci. Lett. 291, 105-108 (2000).

54. J. Steinbrink, F.C. Kempf, A. Villringer, and H. Obrig, "The fast optical signal-robust or elusive when non-invasively measured in the human adult?", Neuroimage 26, 996-1008 (2005).

55. M. Wolf, U. Wolf, J.H. Choi, R. Gupta, L.P. Safonova, L.A. Paunescu, A. Michalos, and E. Gratton, "Functional frequency-domain near-infrared spectroscopy detects fast neuronal signal in the motor cortex", Neuroimage 17, 1868-1875 (2002).

56. M. Wolf, U. Wolf, J.H. Choi, V. Toronov, L.A. Paunescu, A. Michalos, and E. Gratton, "Fast cerebral functional signal in the 100-ms range detected in the visual cortex by frequency-domain near-infrared spectrophotometry", Psychophysiol. 40, 521-528 (2003).

57. G. Morren, U. Wolf, P. Lemmerling, M. Wolf, J.H. Choi, E. Gratton, L. De Lathauwer, and S. Van Huffel, "Detection of fast neuronal signals in the motor cortex from functional near infrared spectroscopy measurements using independent component analysis", Med. Biol. Eng. Comput. 42, 92-99 (2004).

58. M.A. Franceschini and D.A. Boas, "Noninvasive measurement of neuronal activity with near-infrared optical imaging", Neuroimage 21, 372-386 (2004).

59. A.V. Medvedev, S. Borisov, J. Kainerstorfer, R. Guyonneau, M. Riesenhuber, and J. VanMeter, "Event related fast optical signal in an object deteaction taks: A NIRS/EEG comparison study", $13^{\text {th }}$ Annual Meeting of the Organization for Human Brain Mapping 32M, Chicago, 2007.

60. C.Y. Tse, C.L. Lee, J. Sullivan, S.M. Garnsey, G.S. Dell, M. Fabiani, and G. Gratton, "Imaging cortical dynamics of language processing with the event-related optical signal", Proc. Natl. Acad. Sci. USA 104, 17157-17162 (2007).

61. J.K. Thompson, M.R. Peterson, and R.D. Freeman, "Single-neuron activity and tissue oxygenation in the cerebral cortex", Science 299, 1070-1072 (2003).

62. M.M. Plichta, M.J. Herrmann, C.G. Baehne, A.C. Ehlis, M.M. Richter, P. Pauli, and A.J. Fallgatter, "Event-related functional near-infrared spectroscopy (fNIRS): are the measurements reliable?", Neuroimage 31, 116-124 (2006).

63. T. Kono, K. Matsuo, K. Tsunashima, K. Kasai, R. Takizawa, M.A. Rogers, H. Yamasue, T. Yano, Y. Taketani, and N. Kato, "Multiple-time replicability of near-infrared spectroscopy recording during prefrontal activation task in healthy men", Neurosci. Res. 57, 504-512 (2007).

64. B.W. Zeff, B.R. White, H. Dehghani, B.L. Schlaggar, and J.P. Culver, "Retinotopic mapping of adult human visual cortex with high-density diffuse optical tomography", Proc. Natl. Acad. Sci. USA 104, 12169-12174 (2007). 
65. D.A. Boas, A.M. Dale, and M.A. Franceschini, "Diffuse optical imaging of brain activation: approaches to optimizing image sensitivity, resolution, and accuracy", Neuroimage 23, Suppl. 1, S275-S2788 (2004).

66. J.H. Meek, C.E. Elwell, M.J. Khan, J. Romaya, J.S. Wyatt, D.T. Delpy, and S. Zeki, "Regional changes in cerebral haemodynamics as a result of a visual stimulus measured by near infrared spectroscopy", Proc. Biol. Sci. 261, 351-356 (1995).

67. Y. Hoshi, S. Kohri, Y. Matsumoto, K. Cho, T. Matsuda, S. Okajima, and S. Fujimoto, "Hemodynamic responses to photic stimulation in neonates", Pediatr. Neurol. 23, 323-327 (2000).

68. T. Karen, G. Morren, D. Haensse, A.S. Bauschatz, H.U. Bucher, and M. Wolf, "Hemodynamic response to visual stimulation in newborn infants using functional near-infrared spectroscopy", Hum. Brain Mapp. 29, 453-460 (2008).

69. S. Chen, K. Sakatani, W. Lichty, P. Ning, S. Zhao, and H. Zuo, "Auditory-evoked cerebral oxygenation changes in hypoxic-ischemic encephalopathy of newborn infants monitored by near infrared spectroscopy", Early Hum. Dev. 67, 113-121 (2002).

70. K. Sakatani, S. Chen, W. Lichty, H. Zuo, and Y.P. Wang, "Cerebral blood oxygenation changes induced by auditory stimulation in newborn infants measured by near infrared spectroscopy", Early Hum. Dev. 55, 229-236 (1999).

71. P. Zaramella, F. Freato, A. Amigoni, S. Salvadori, P. Marangoni, A. Suppjei, B. Schiavo, and L. Chiandetti, "Brain auditory activation measured by near-infrared spectroscopy (NIRS) in neonates", Pediatr. Res. 49, 213-219 (2001).

72. K. Kotilahti, I. Nissil, R. Makela, T. Noponen, L. Lipiainen, N. Gavrielides, T. Kajava, M. Huotilainen, V. Fellman, P. Merilainen, and T. Katila, "Near-infrared spectroscopic imaging of stimulus-related hemodynamic responses on the neonatal auditory cortices", Proc. SPIE 5693, 388-395 (2005).

73. Y. Saito, T. Kondo, S. Aoyama, R. Fukumoto, N. Konishi, K. Nakamura, M. Kobayashi, and T. Toshima, "The function of the frontal lobe in neonates for response to a prosodic voice", Early Hum. Dev. 83, 225-230 (2007).
74. M. Bartocci, J. Winberg, C. Ruggiero, L.L. Bergqvist, G. Serra, and H. Lagercrantz, "Activation of olfactory cortex in newborn infants after odor stimulation: a functional near-infrared spectroscopy study", Pediatr. Res. 48, 18-23 (2000).

75. M. Bartocci, J. Winberg, G. Papendieck, T. Mustica, G. Serra, and H. Lagercrantz, "Cerebral hemodynamic response to unpleasant odors in the preterm newborn measured by near-infrared spectroscopy", Pediatr. Res. 50, 324-330 (2001).

76. M. Bartocci, L.L. Bergqvist, H. Lagercrantz, and K.J. Anand, "Pain activates cortical areas in the preterm newborn brain", Pain 122, 109-117 (2006).

77. H.U. Bucher, T. Moser, K. von Siebenthal, M. Keel, M. Wolf, and G. Duc, "Sucrose reduces pain reaction to heel lancing in preterm infants: a placebo-controlled, randomized and masked study", Pediatr. Res. 38, 332-335 (1995).

78. R. Slater, S. Boyd, J. Meek, and M. Fitzgerald, "Cortical pain responses in the infant brain", Pain 123, 332-334 (2006).

79. J.H. Meek, M. Firbank, C.E. Elwell, J. Atkinson, O. Braddick, and J.S. Wyatt, "Regional hemodynamic responses to visual stimulation in awake infants", Pediatr. Res. 43, 840-843 (1998).

80. G. Taga, K. Asakawa, A. Maki, Y. Konishi, and H. Koizumi, "Brain imaging in awake infants by near-infrared optical topography", Proc. Natl. Acad. Sci. USA 100, 10722-10727 (2003).

81. S.R. Hintz, D.A. Benaron, A.M. Siegel, A. Zourabian, D.K. Stevenson, and D.A. Boas, "Bedside functional imaging of the premature infant brain during passive motor activation", J. Perinat. Med. 29, 335-343 (2001).

82. M. Pena, A. Maki, D. Kovacic, G. Dehaene-Lambertz, H. Koizumi, F. Bouquet, and J. Mehler, "Sounds and silence: an optical topography study of language recognition at birth", Proc. Natl. Acad. Sci. USA 100, 11702-11705 (2003).

83. K. Isobe, T. Kusaka, K. Nagano, K. Okubo, S. Yasuda, M. Kondo, S. Itoh, and S. Onishi, "Functional imaging of the brain in sedated newborn infants using near infrared topography during passive knee movement", Neurosci. Lett. 299, 221-224 (2001). 